\title{
Profile of diabetic ketoacidosis at a teaching hospital in Benghazi, Libyan Arab Jamahiriya
}

R.R. Elmehdawi and H.M. Elmagerhei ${ }^{2}$

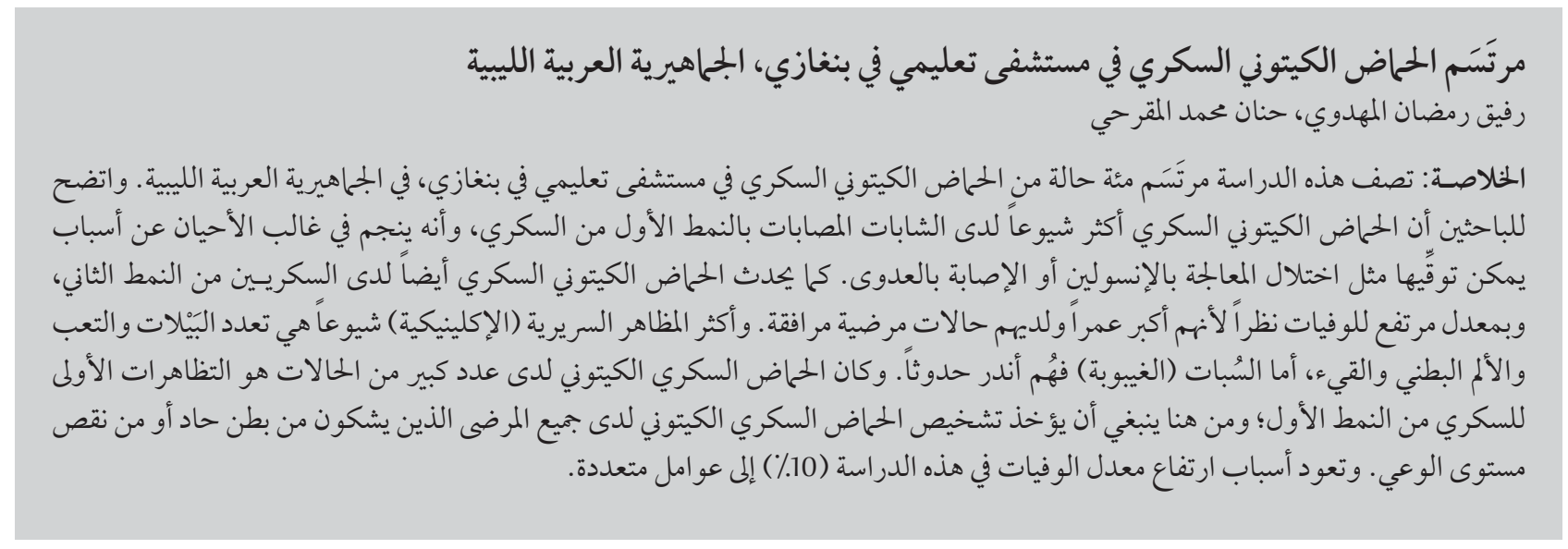

ABSTRACT This study described the profile of 100 cases of diabetic ketoacidosis (DKA) at a teaching hospital in Benghazi, Libyan Arab Jamahiriya. DKA was more frequent in young women with type 1 diabetes and mostly due to preventable causes, e.g. disrupted insulin treatment and/or infection. DKA also occurred in type 2 diabetics, with a higher mortality rate, as they were older patients with co-morbidity. Polyurea, fatigue, abdominal pain and vomiting were the most common clinical features, while coma was rarer. A high number of cases were first presentations of type 1 diabetes; hence this diagnosis should be considered in all patients with acute abdomen or decreased level of consciousness. The reasons for high mortality rate in this study (10\%) were multifactorial.

\section{Profil de l'acidocétose diabétique dans un hôpital universitaire de Benghazi (Jamahiriya arabe libyenne)}

RÉSUMÉ Cette étude décrit le profil de 100 cas d'acidocétose diabétique dans un hôpital universitaire de Benghazi (Jamahiriya arabe libyenne). L'acidocétose diabétique était plus fréquente chez les jeunes femmes souffrant de diabète de type 1, et était essentiellement due à des causes telles qu'une interruption de l'insulinothérapie et/ou une infection. Elle a également été observée chez des personnes souffrant de diabète de type 2, avec un taux de mortalité plus élevé, les patients étant plus âgés et présentant une comorbidité. Les principales manifestations cliniques étaient une polyurie, un état de fatigue, des douleurs abdominales et des vomissements, le coma étant plus rare. Un grand nombre de cas étaient des premières présentations de diabète de type 1. C'est pourquoi ce diagnostic devrait être envisagé chez tous les patients présentant un abdomen aigu ou une altération de la conscience. Les causes du taux de mortalité élevé dans cette étude (10\%) étaient multifactorielles. 


\section{Introduction}

Type 1 diabetes mellitus (DM) is characterized by the development of a state of complete insulin deficiency which makes patients prone to ketoacidosis. Diabetic ketoacidosis (DKA) is a frequent cause of morbidity and mortality in type 1 diabetes patients. However, it also occurs occasionally in patients with type $2 \mathrm{DM}$ during severe stress, for example sepsis and myocardial infarction $[1-5]$.

About $12.8 \%$ of the diabetic adults in Benghazi have type $1 \mathrm{DM}[6]$. The incidence of type $1 \mathrm{DM}$ in the Libyan population up to 34 years old is estimated to be approximately 9 per 100000 per year [7]. DKA is the presenting feature of about $30 \%$ of Libyan children with type $1 \mathrm{DM}[8]$. However, the annual incidence of DKA in Libyan diabetics is not known. Even so, the disorder seems to contribute appreciably to the number of hospital admissions as it constitutes 9\% of all diabetes-related admissions [9] and $3.2 \%$ of all acute admissions to the medical intensive care unit in Benghazi [10]. A report from Benghazi revealed that $1.6 \%$ of the adult diabetic patients had been admitted to hospital in the previous year because of DKA [6]. The overall mortality rate from DKA in the Libyan Arab Jamahiriya ranges from $2 \%$ to $9.4 \%$ of all DKA admissions according to different reports $[1,2,11]$. Although many DKA-associated deaths are due to concomitant illnesses (e.g. myocardial infarction, septicaemia), others are preventable and are due to delay in presentation, diagnosis and/or errors in management.

The aim of this study was to determine the pattern of DKA at a teaching hospital in Benghazi, Libyan Arab Jamahiriya, to assess the clinical and biochemical characteristics of the patients and the precipitating factors for DKA, and to correlate these parameters with the outcome.

\section{Methods}

A descriptive cross-sectional retrospective analysis was made of the records of adult patients admitted to the Seventh of October teaching hospital, Benghazi, and diagnosed with DKA between January 2003 and December 2006. During this 4-year period 200 suspected DKA cases were admitted to the medical ward and intensive care unit of the hospital. Out of these, we included only 100 episodes in this study because for the other 100 the records were missing important information.

The following variables were recorded: age, sex, nationality, duration of diabetes, precipitating factors for DKA, duration of symptoms before seeking medical advice, clinical features, laboratory findings at presentation with DKA (plasma glucose, serum blood urea nitrogen, serum creatinine, serum sodium and potassium, urine acetone, arterial blood $\mathrm{pH}$, plasma bicarbonate and plasma osmolarity), associated complications, duration of hospitalization and outcome. Patients were classified as having type 1 or type 2 diabetes based on their treatment history.

The criteria used to diagnose DKA in the patients included in the study were: typical clinical features such as hyperventilation, vomiting and dehydration; hyperglycaemia $\geq 250 \mathrm{mg} / \mathrm{dL}$; arterial blood $\mathrm{pH} \leq 7.3$ and/or serum bicarbonate $\leq 18 \mathrm{mmol} / \mathrm{L}$; and presence of ketonuria (2+ on dipstick).

The severity of DKA was defined according to the American Diabetes Association (ADA) criteria [12,13] as mild (arterial blood pH 7.25-7.30), moderate ( $\mathrm{pH} 7.00-7.24)$ or severe $(\mathrm{pH}<7.00)$. Effective osmolarity was calculated using the ADA formula: [(2 $x$ sodium $)+$ plasma glucose $/ 18]$, with normal values being $290 \mathrm{mmol} / \mathrm{kg}$ water. Blood urea nitrogen was not included in this calculation because urea has less osmotic activity [14].
The data were analysed using SPSS, version 11.0. Differences between groups were tested statistically using the chi-squared test and independent samples $t$-test. Differences were considered statistically significant at $P$-value $<0.05$.

\section{Results}

We studied 100 episodes of DKA in 81 patients; 8 patients were responsible for 19 episodes (3 patients had 3 episodes and 5 patients had 2 episodes). These 100 admissions accounted for 769 medical and intensive care bed-days (2.4\% of the total medical and intensive care bed-days). According to the ADA criteria, 29 of the episodes were mild, 37 were moderate and 34 were severe. Type $1 \mathrm{DM}$ was the diagnosis in 90 of the episodes, while the other 10 had type 2. There were 19 episodes in newly diagnosed cases and 81 episodes in previously known cases of DM.

\section{Patient and disease characteristics}

A higher number of episodes were in females (71) than males (29) (female to male ratio 2.3:1) (Table 1). The age distribution is shown in Figure 1. The mean age of all patients was 29.2 years (range 15-68 years); males were slightly older than females [mean age 32.0 (SD 14.9) years versus 28.4 (SD 9.8) years], but this difference was not statistically significant.

Patients with type 2DMwere signifcantly older than those with type $1 \mathrm{DM}$ (mean age 52.6 versus 26.5 years) $(P<$ 0.001 ) (Table 1). Previously diagnosed patients were also older on average than the new cases of DM (mean age 30.1 versus 25.1 years), but the difference was not significant (Table 1).

\section{Clinical features at admission}

Disruption of insulin treatment (complete stoppage or reduction of insulin dose or frequency) was the commonest precipitating factor for the episode of 


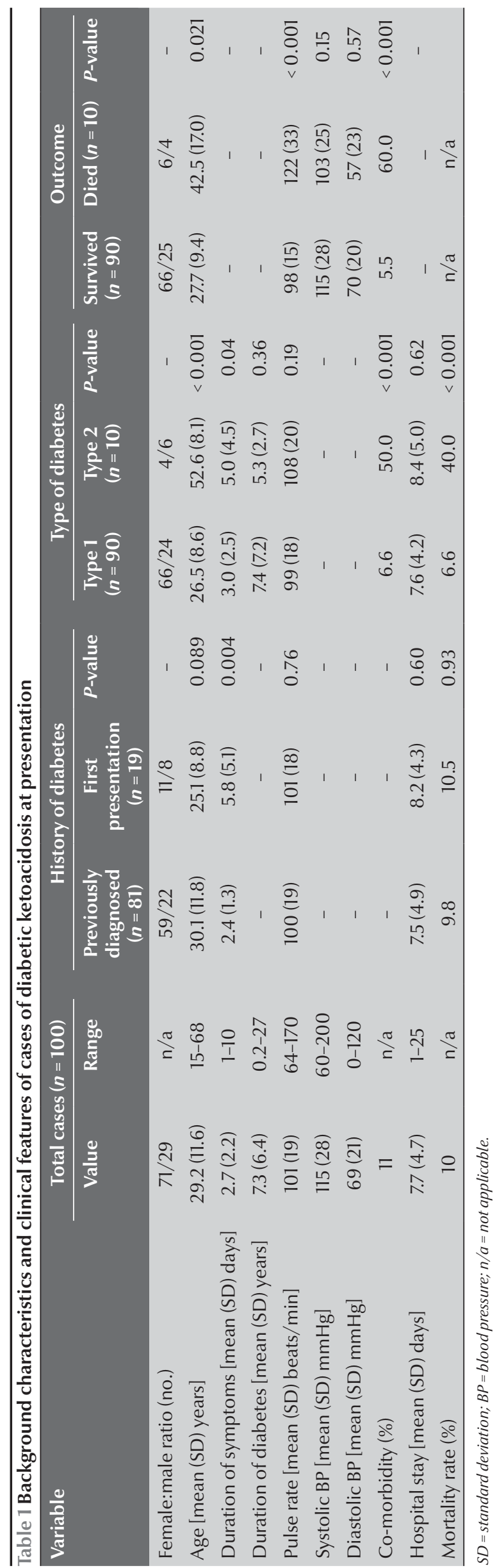

DKA in 38 cases. Infection was the next most common reason in 30 cases: urinary tract infection (13 cases), upper respiratory tract infection (8), lower respiratory tract infection (3), abscess (2) and gastroenteritis, tuberculosis, diabetic foot, peritonitis ( 1 case each). In 19 cases DKA was the first manifestation of diabetes. Other causes were rarer: myocardial infarction ( 2 cases), emotional stress (2) and trauma (1). In 3 cases no obvious precipitating factor could be identified and data were missing for 5 episodes.

The commonest presenting symptoms in this study were general weakness and fatigue (100 cases), polyurea and polydipsia (100), abdominal pain nausea and/or vomiting (64). Other symptoms were drowsiness (15 cases), dyspnoea (12), coma (5), headache (3) and haematemesis (2). Table 1 shows the haemodynamic features at admission.

The mean duration of symptoms before presentation to the hospital was 2.7 (SD 2.2) days (range 1-10 days) (Table 1 ), with $48 \%$ of the cases presenting within the same day of the onset of symptoms and $62 \%$ within 48 hours. The duration of symptoms was significantly longer in patients with type $2 \mathrm{DM}$ as compared with type $1 \mathrm{DM}(P<0.05)$ (Table 1). Also the mean duration of symptoms was longer in new cases of DM as compared with the previously diagnosed cases (Table 1). The mean duration of symptoms was significantly longer in males than females [4.0 (SD 3.7) days versus 2.8 (SD 2.3) days] ( $P$ $<0.05$ ).

\section{Biochemical abnormalities at admission}

Table 2 summarizes the biochemical parameters of DKA cases at presentation. Plasma glucose levels were higher, but not significantly so, in cases presenting for the first time than in those with a previous diagnosis (Table 2). Serum sodium was significantly higher in the new cases of DM than the previously diagnosed cases $(P<0.05)$ (Table 2$)$. Sodium levels were also significantly higher in males than in females [mean 137.9 (SD 13) versus $136.9(\mathrm{SD} 8) \mathrm{mmol} / \mathrm{L}](P<$ $0.05)$. Serum potassium at admission was significantly lower in patients who subsequently died than in the survivors $(P<$ $0.05)$ (Table 2). Blood urea nitrogen was higher for those with type $2 \mathrm{DM}$ than type 1 and in the new cases compared with previously diagnosed cases (Table 2) but these differences were not significant. Serum creatinine was raised in $91 \%$ of cases at admission.

\section{Complications and outcome}

The mean hospital stay was 7.7 days, range 1-25 days (Table 1).

About $66 \%$ of the cases had some sort of complication. Some of these were due to the episode of DKA itself, e.g. coma ( 5 cases), hypokalaemia (serum potassium $<3.5 \mathrm{mmol} / \mathrm{L}$ ) (13 cases). However, some complications developed during management due to improper therapeutic interventions, 


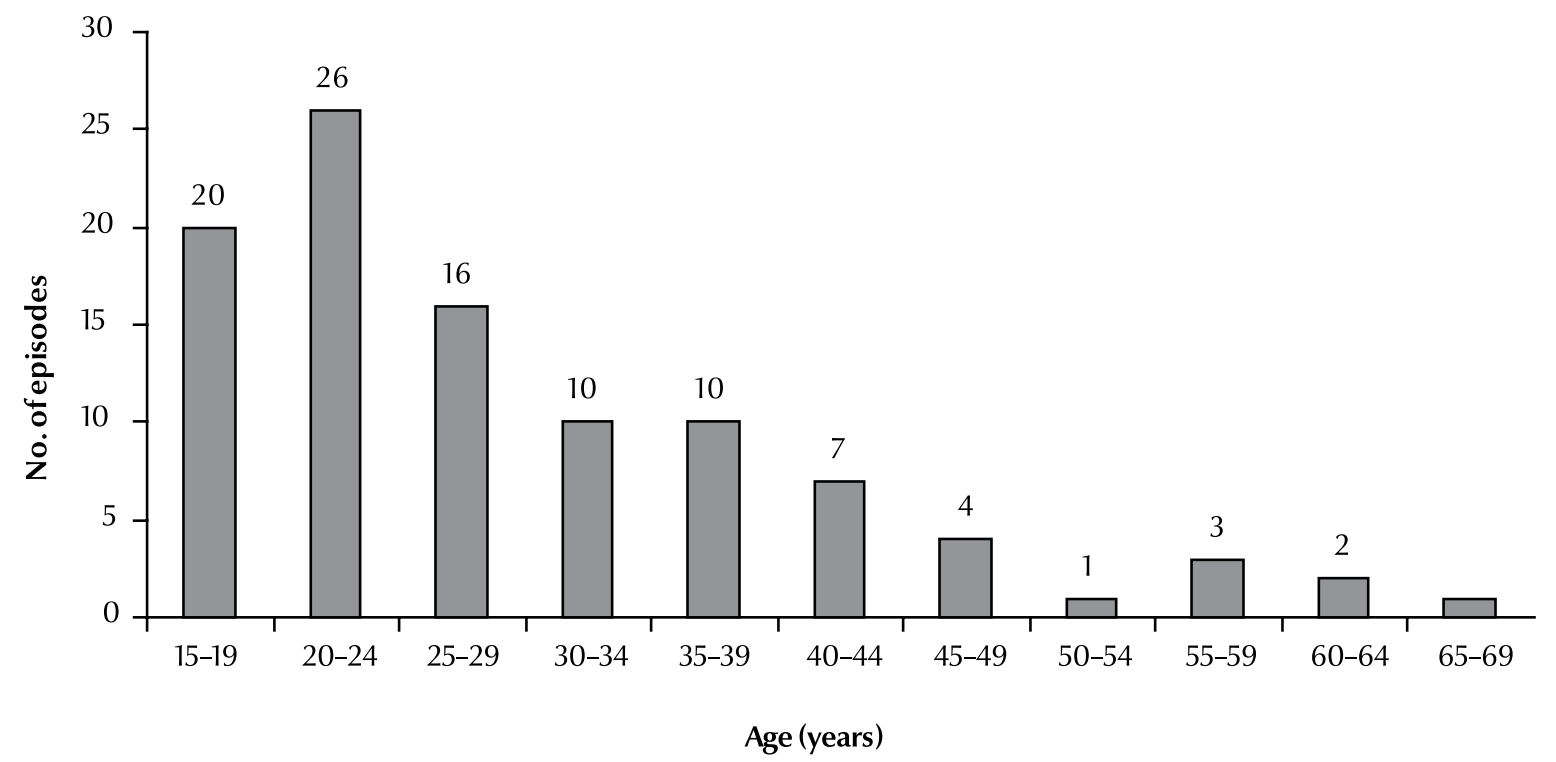

Figure 1 Age distribution of episodes of diabetic ketoacidosis $(n=100)$

e.g. pneumothorax (1 case) and hypokalaemia from treatment (3 cases). The most common complications encountered during DKA were prerenal azotaemia (urea $>40 \mathrm{mmol} / \mathrm{L})(28$ cases) and electrolyte disturbances, e.g. hypokalaemia (16), hyperkalaemia (13) and hypernatraemia (serum sodium > $145 \mathrm{mmol} / \mathrm{L})(10)$. More serious complications were rare: e.g. upper gastrointestinal bleeding (2 cases), acute gastric dilatation (1), acute renal failure (1 case), adult respiratory distress syndrome (1) and disseminated intravascular coagulation (1).

There were 10 deaths among the DKA cases (10\% of episodes, $12.3 \%$ of patients) (4 males and 6 females), and the mortality rate among males was slightly higher than females (13.8\% versus $8.4 \%$ ). The mean age of females who died was significantly lower than the males $[37$ (SD 17) years versus 51 (SD 16) years] $(P<0.001)$. The mortality rate was significantly higher among patients with type 2 DM than those with type 1 (40.0\% versus $6.6 \%$ ) $(P<0.001)$ (Table 1$)$. Regarding the severity of DKA, there were 8 deaths (23.5\%) among the severe cases and 2 deaths (5.4\%) among the moderate
DKA cases; none of the patients with mild DKA died.

An important comorbidity was associated with 6 of the 10 deaths: myocardial infarction (2 cases), acute abdomen, pulmonary tuberculosis, acute renal failure or diabetic foot (1 case each). The following parameters were associated with a significantly increased mortality: age $>40$ years, type 2 DM, depressed level of consciousness at presentation, comorbidity, pulse rate $\geq 115$ beats $/ \mathrm{min}$, systolic blood pressure $\leq 105 \mathrm{mmHg}$, diastolic blood pressure $\leq 65 \mathrm{mmHg}$, plasma glucose $\geq 525 \mathrm{mg} / \mathrm{dL}$, serum sodium $\geq 144$ $\mathrm{mmol} / \mathrm{L}$, blood urea nitrogen $\geq 50 \mathrm{mg} /$ $\mathrm{dL}$, serum creatinine $\geq 4 \mathrm{mg} / \mathrm{dL}$, arterial blood $\mathrm{pH} \leq 7$ and plasma osmolality $\geq$ $325 \mathrm{mOsm} / \mathrm{kg}$ water (Table 3).

\section{Discussion}

Despite the small sample size of this study, and the fact that it was a retrospective analysis conducted at a teaching hospital, all of which are important limitations, this study gives a basic profile of DKA in this part of the world where studies about DKA are few.

\section{Patient and disease characteristics}

While most of the episodes of DKA in this study were due to type $1 \mathrm{DM}, 10 \%$ were due to type $2 \mathrm{DM}$; this compares with 15\% reported by Roaeid from the same hospital between 1997 and 1999 [1] and $11.6 \%$ reported by El-Sharief in another Libyan study from Tripoli [2]. Although DKA is usually considered as a unique marker for type $1 \mathrm{DM}$ these reports clearly demonstrate that it can occur among type 2 diabetic patients. This fact is increasingly recognized throughout the world [3-5].

Females were $70 \%$ of all DKA admissions. A female predominance was previously reported in Benghazi by Roaeid and Kablan [1], whereas ElSharief reported a nearly equal ratio of sexes in Tripoli [2]. This discrepancy in the sex ratio between different cohorts was also observed in studies from Saudi Arabia [15] and Jordan [16]. We think that the cause of these variations is multifactorial, including environmental, genetic and social differences between the cohorts. However, the small study samples and errors in sampling could be important contributing factors. The female predominance was maintained 


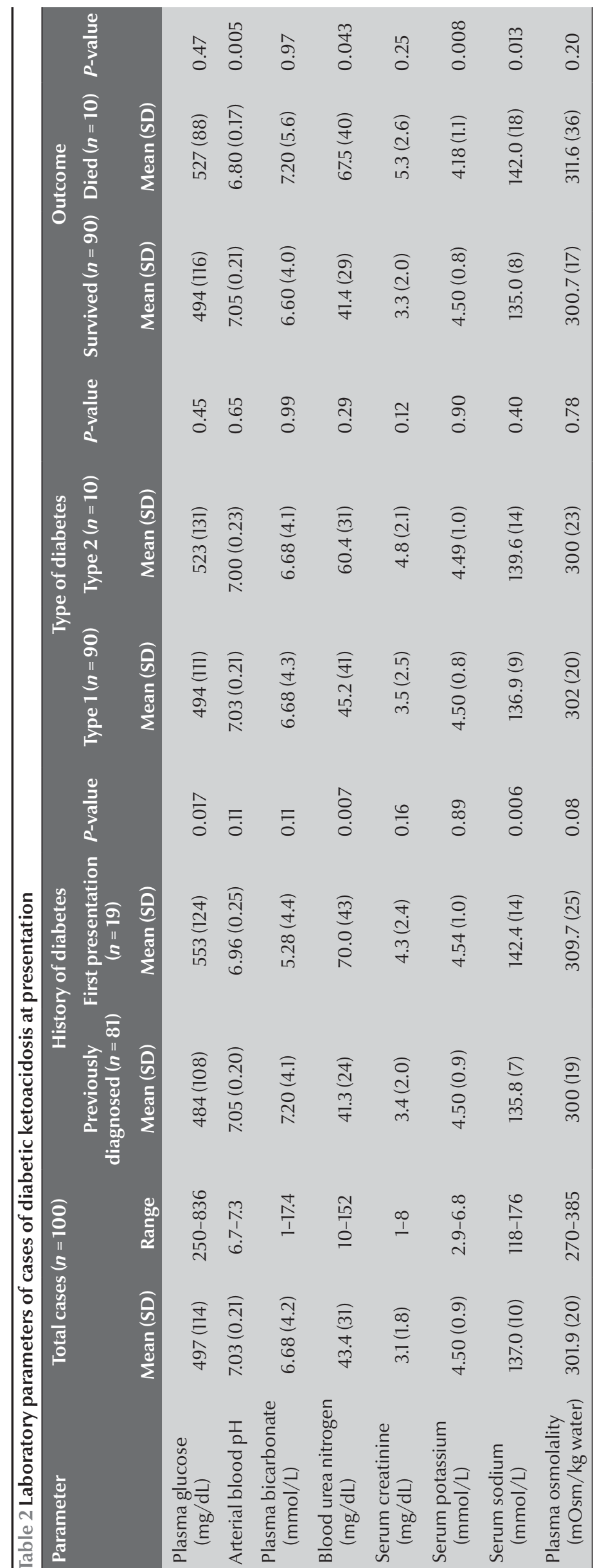

in the group of known diabetics, among the new cases and in those with type $1 \mathrm{DM}$. However, this predominance was reversed in patients with type $2 \mathrm{DM}$, where males constituted $60 \%$ of the cases.

The mean age of all patients ( 29.2 years) is similar to that previously reported by Roaeid and Kablan (28.9years) [1]. Males were slightly older than females, although this was not statistically significant. However, patients with type 2 DM were significantly older than those with type 1 (52.6 and 26.5 years respectively) presumably because type $2 \mathrm{DM}$ tends to develop in adults older than 40 years while type $1 \mathrm{DM}$ usually occurs at a much younger age.

\section{Clinical features at admission}

Poor drug compliance was the major precipitating factor of DKA in our patients (38\%); which is similar to El-Shariefs report from Tripoli (36.7\%) [2], but lower than 54.4\% from Saudi Arabia [15]. However, it worth mentioning that the rate of DKA due to treatment disruption in the current study (from 2003-06) is much lower than in the previous study in Benghazi from the years 1997-99 (64\%) [1]; this might indicate an improvement in patients' compliance and education. Infections were the second most common precipitating factor (30\%), similar to other reports from Benghazi (29.7\%) [1], Tripoli (21.7\%) [2] and Saudi Arabia (28\%) [15]. In developed countries infection is the commonest precipitating factor of DKA, while insulin disruption ranks second, perhaps due to better patient education [17]. Cases of DKA caused by drug omission and infection are potentially preventable with good patient education and proper outpatient management. About 3\% of our patients had no obvious precipitating factor, which is in concordance with $2 \%-10 \%$ reported from other parts of the world [18].

The mean duration of symptoms before presentation to the hospital was 2.7 days, with $48 \%$ of the patients presenting within the same day of the onset of symptoms, and this probably contributed to the low rate of serious clinical features at presentation. However, the duration was longer in those who were not known diabetics ( 5.8 versus 2.7 days), because patients may not realize what is happening at the first episode of DKA. The duration of symptoms 


\begin{tabular}{|c|c|c|}
\hline Variable & Mortality (\%) & $P$-value \\
\hline \multicolumn{3}{|l|}{ Age (years) } \\
\hline$\geq 40$ & 27.7 & $<0.001$ \\
\hline$<40$ & 6.0 & \\
\hline \multicolumn{3}{|l|}{ Sex } \\
\hline Male & 13.8 & 0.46 \\
\hline Female & 8.5 & \\
\hline \multicolumn{3}{|l|}{ Type of diabetes } \\
\hline Type 2 & 40.0 & $<0.001$ \\
\hline Type 1 & 6.6 & \\
\hline \multicolumn{3}{|l|}{ Co-morbidity } \\
\hline Yes & 50.0 & $<0.001$ \\
\hline No & 4.5 & \\
\hline \multicolumn{3}{|c|}{ Level of consciousness } \\
\hline Unconscious & 35.0 & $<0.001$ \\
\hline Fully conscious & 3.8 & \\
\hline \multicolumn{3}{|l|}{ Pulse (beats/min) } \\
\hline$\geq 115$ & 22.7 & 0.024 \\
\hline$<115$ & 7.4 & \\
\hline \multicolumn{3}{|c|}{ Blood pressure $(\mathrm{mmHg})$} \\
\hline Systolic $\leq 105$ & 21.6 & 0.005 \\
\hline Systolic $>105$ & 3.5 & \\
\hline Diastolic $\leq 65$ & 22.5 & 0.008 \\
\hline Diastolic $>65$ & 4.8 & \\
\hline \multicolumn{3}{|c|}{ Plasma glucose (mg/dL) } \\
\hline$\geq 525$ & 12.0 & 0.04 \\
\hline$<525$ & 8.6 & \\
\hline \multicolumn{3}{|l|}{ Arterial blood $\mathrm{pH}$} \\
\hline$\leq 7$ & 16.3 & $<0.001$ \\
\hline$>7$ & 3.9 & \\
\hline \multicolumn{3}{|c|}{ Blood urea nitrogen $(\mathrm{mg} / \mathrm{dL})$} \\
\hline$\geq 50$ & 24.0 & 0.012 \\
\hline$<50$ & 5.5 & \\
\hline \multicolumn{3}{|c|}{ Serum creatinine $(\mathrm{mg} / \mathrm{dL})$} \\
\hline$\geq 4$ & 24.1 & 0.039 \\
\hline$<4$ & 4.9 & \\
\hline \multicolumn{3}{|c|}{ Serum sodium $(\mathrm{mmol} / \mathrm{L})$} \\
\hline$\geq 144$ & 28.5 & 0.02 \\
\hline$<144$ & 7.6 & \\
\hline \multicolumn{3}{|c|}{ Plasma osmolality (mOsm/kg water) } \\
\hline$\geq 325$ & 44.0 & 0.018 \\
\hline$<325$ & 7.1 & \\
\hline
\end{tabular}

was significantly longer for those with type $2 \mathrm{DM}$ than type $1 \mathrm{DM}$ because these patients are not primarily ketosisprone but ketoacidosis may develop slowly due to secondary insulin deficiency which builds up gradually as a result of glucose toxicity. The duration of symptoms was also significantly longer in males than in females, suggesting that males postpone medical consultation longer than females.

Fatigue, polyurea, abdominal pain and vomiting were the most common presenting symptoms in our study, which is similar to the report from Tripoli [2]. Only 5\% of our patients had coma at presentation compared with $25 \%$ reported from a study in Nairobi [19], perhaps due to ethnic differences in diabetes severity or better education among our patients and easier availability of immediate health care services in our country which could contribute to earlier presentation and hence less severe clinical features.

\section{Biochemical abnormalities}

The mean plasma glucose level was higher in the new cases of DM and in those with type $2 \mathrm{DM}$, probably because patients belonging to these groups presented 2 to 3 days later than the known cases of type $1 \mathrm{DM}$, allowing a higher degree of hyperglycaemia to develop. The same was true for the higher serum sodium and blood urea in these groups, which indicates a more severe dehydration due to longer duration of glucose-mediated osmotic diuresis and water loss in excess of electrolytes. The great majority of patients (75\%) were eukalaemic at presentation, 13\% were hypokalaemic, while another 3\% developed hypokalaemia as a result of potassium under-replacement during management. Hyperkalaemia was nearly as common as hypokalaemia at admission. The raised serum creatinine seen in most of the DKA patients was likely a false elevation, as acetoacetate artificially raises measured creatinine in the standard colorimetric assay $[20,21]$. Metabolism of the acetoacetate following the administration of insulin will lower the measured serum creatinine concentration towards its true value.

\section{Outcome}

The mean hospital stay was 7.7 days, with some cases discharged on their 
request as early as the first day of admission and a few others who stayed at hospital more than 2 weeks for treating the precipitating factors and complications or for social reasons. This is similar to the average length of stay in other reports from the Libyan Arab Jamahiriya $[1,2]$. However, it is longer than the average length of stay for DKA in the United States (3.6 days) [22] and also longer than in southern Jordan where the average length of stay was 3.4 days [16]. Our longer hospitalization will result in higher costs of DKA management.

Fortunately, despite the high rate of DKA-related complications (68\%) most of the complications were not serious and were easy to treat, e.g. mild electrolyte disturbances and prerenal azotaemia. However, serious complications were encountered in some cases, e.g. severe electrolyte disturbances. Unfortunately, some of the complications were iatrogenic, e.g. such as pneumothorax due to insertion of a central venous line and hypokalaemia due to potassium under-correction.

Despite the low rate of serious complications, the mortality rate of our DKA cases was high (10\%). While this is similar to the report from Tripoli (9.4\%) [2], it is much higher than the rate that was previously reported in Benghazi (3.8\%) [1], which might indicate a deficiency in diagnostic and therapeutic facilities at hospitals or a decline in doctors' and co-workers' skills and experience in the management of DKA. The mortality rate was also higher than figures from Saudi Arabia [15] and Jordan [16] (2.9\% and $4.8 \%$ respectively); however, it was better than from Nairobi, where the mortality rate was nearly 30\% [19]. This high variation in the mortality rates may reflect variability in access to medical services and standards of inpatient management as well as patient education.

DKA patients with type 2 DM had a significantly higher mortality rate (40.0\%) than patients with type $1 \mathrm{DM}$ (6.6\%), probably because type $2 \mathrm{DM}$ patients were significantly older and more likely to have comorbidity. Overall, patients who died were significantly older, with more comorbidity, a lower level of consciousness at presentation, lower blood pressure, faster pulse rate, higher plasma glucose, higher serum sodium, higher serum creatinine, higher plasma osmolality and lower blood $\mathrm{pH}$.

\section{Conclusion}

DKA was more common in Libyan females than in males. It was frequently seen in young women with type $1 \mathrm{DM}$, mostly caused by potentially preventable causes such as disruption of insulin treatment and/or infection. DKA also occurred in type 2 DM patients and when this happened it resulted in higher mortality as it occurred in older persons with coexisting morbidity.

Polyurea, fatigue, abdominal pain and vomiting were the most common clinical features in our patients, while coma was relatively uncommon. DKA as a first presentation of type $1 \mathrm{DM}$ accounted for a considerable number of cases; hence this diagnosis should be considered in all patients with acute abdomen or decreased level of consciousness, even if they are not known diabetics.

The high mortality rate in this study ( $10 \%$ of episodes) was due to multifactorial reasons, partly patient-related factors such as old age, and comorbidity, and partly disease-related factors such as DKA severity. However some may have been due to therapy-related factors such as errors in management. Further studies are needed to clarify the factors behind this high mortality rate and steps to reduce the rate should be taken.

\section{References}

1. Roaeid R, Kablan A. Diabetic ketoacidosis in Benghazi characteristics and outcome in 211 patients. Garyounis medical journal, 2004, 21(1):11-4.

2. El-Sharief HJ. Diabetic ketoacidosis: Tripoli Medical Center experience. Jamahiriya medical journal, 2006, 5 (1):51-4.

3. Seyoum B, Berhanu P. Profile of diabetic ketoacidosis in a predominantly African American urban patient population. Ethnicity and disease, 2007, 17(2):234-7.

4. Newton CA, Raskin P. Diabetic ketoacidosis in type 1 and type 2 diabetes mellitus: clinical and biochemical differences. $A r$ chives of internal medicine, 2004. 27, 164 (17):1925-31.

5. Umpierrez GE, Smiley D, Kitabchi AE. Narrative review: ketosisprone type 2 diabetes mellitus. Archives of internal medicine, 2006, 144(5):350-7.

6. Roaeid RB, Kablan AA. Profile of diabetes health care at Benghazi Diabetes Centre, Libyan Arab Jamahiriya. Eastern Mediterranean health journal, 2007, 13(1):168-76.
7. Kadiki OA, Reddy MR, Marzouk AA. Incidence of insulin-dependent diabetes (IDDM) and non-insulin-dependent diabetes (NIDDM) (0-34 years at onset) in Benghazi, Libya. Diabetes research and clinical practice, 1996, 32(3):165-73.

8. Kadiki OA, Gerryo SE, Khan MM. Childhood diabetes mellitus in Benghazi (Libya). Tropical pediatrics, 1987, 33(3):136-9.

9. Roaeid RB. Hospital admissions of diabetic patients in Benghazi. Diabetes international, 2002, 12 (1):24-5.

10. Roaeid RB et al. Admission patterns and outcome in an adult medical intensive care unit in Benghazi. Garyounis medical journal, 2005, 22(1):61-6.

11. Lakhdar AA, Elharboush S. Characteristics and outcome of ketoacidosis in Libyan diabetic patients. Diabetes international, 1999, 16(6):171-3.

12. Kitabchi AE et al. Hyperglycemic crises in adult patients with diabetes: a consensus statement from the American Diabetes Association. Diabetes care, 2006, 29 (12):2739-48. 
13. Kitabchi AE et al. Hyperglycemic crises in diabetes. Diabetes care, 2004, 27(Suppl. 1):S94-102.

14. Siperstein MD. Diabetic ketoacidosis and hyperosmolar coma. Endocrinology and metabolism clinics of North America, 1992, 21(2):415-32.

15. Qari FA. Precipitating factors for diabetic ketoacidosis. Saudi medical journal, 2002, 23(2):173-6.

16. Tahboub I, Shalan JB. Diabetic ketoacidosis in southern Jordan: five-year experience. Eastern Mediterranean health journal, 2000, 6(5-6):1035-8.

17. Wallace TM, Matthews DR. Recent advances in the monitoring and management of diabetic ketoacidosis. Quarterly journal of medicine, 2004, 97(12):773-80.

18. Umpierrez GE, Khajavi M, Kitabchi AE. Review: diabetic ketoacidosis and hyperglycemic hyperosmolar nonke- totic syndrome. American journal of medical science, 1996, 311(5):225-33.

19. Mbugua PK et al. Diabetic ketoacidosis: clinical presentation and precipitating factors at Kenyatta National Hospital, Nairobi. East African medical journal, 2005, 82(12 Suppl.):S191-6.

20. Molitch ME et al. Spurious serum creatinine elevations in ketoacidosis. Annals of internal medicine, 1980, 93(2):280-1.

21. Kemperman FA et al. The influence of ketoacids on plasma creatinine assays in diabetic ketoacidosis. Journal of internal medicine, 2000, 248(6):511-7.

22. Diabetes data and trends. Centers for Disease Control and Prevention [website] (http://www.cdc.gov/Diabetes/statistics/ dkafirst/fig2.htm, accessed 5 September 2009).

\section{Mortality and burden of disease attributable to selected major risks}

Mortality and burden of disease attributable to selected major risks uses a comprehensive framework for studying health risks that was developed for the World Health Report 2002, which presented estimates for the year 2000. The report provides an update for the year 2004 for 24 global risk factors. It uses updated information from WHO programmes and scientific studies for both exposure data and the causal associations of risk exposure to disease and injury outcomes. The burden of disease attributable to risk factors is measured in terms of lost years of healthy life using the metric of the disability-adjusted life year (DALY). The DALY combines years of life lost due to premature death with years of healthy life lost due to illness and disability.

Health risks are in transition: populations are ageing owing to successes against infectious diseases; at the same time, patterns of physical activity and food, alcohol and tobacco consumption are changing. Low- and middle-income countries now face a double burden of increasing chronic, noncommunicable conditions, as well as the communicable diseases that traditionally affect the poor.

Further information about this and other WHO publications can be found at: http://www.who.int/publications/en/ 